\title{
Design and Selection Criteria of High Temperature Accelerometers for Aerospace Propulsion
}

\author{
Bob Metz ${ }^{1}$ \\ PCB Piezotronics, Inc. \\ 3425 Walden Ave., Depew, NY 14043 \\ Co-Author: Hans Ensinger ${ }^{2}$
}

\section{Introduction}

Accelerometers and pressure sensors for measurement in aerospace propulsion systems require special consideration during design and manufacturing processes. Specialized applications frequently require use of a single sensor model, which must be capable of operating over significantly wider temperature ranges, for example, -420 to $+1300{ }^{\circ} \mathrm{F}\left(-251\right.$ to $\left.+705^{\circ} \mathrm{C}\right)$, while providing high accuracy, stability and reliability. Typical applications for high temperature aerospace propulsion sensors include measurement on gas turbine engines both in-flight and in test cells, as well as rocket motors and thruster assemblies. The same sensor might be required to withstand radiation and be used in monitoring vibration inside a nuclear power plant or space vehicle, or the cryogenic conditions of liquid propellants. These environments present a multitude of measurement challenges.

Materials and construction techniques must be optimized, not only to enhance high-temperature performance, but also to allow operation in the presence of gamma and neutron radiation without degradation. Whether used in aircraft engines, space vehicles, or power generation stations, these sensors must provide high levels of accuracy, stability and reliability. Therefore these instruments used in extreme environments such as cryogens and high-temperature require special consideration during the design and manufacturing process. This paper will discuss the design and selection criteria for a new high temperature shear mode accelerometer and its benefits for turbine engine health monitoring.

\section{Nomenclature}

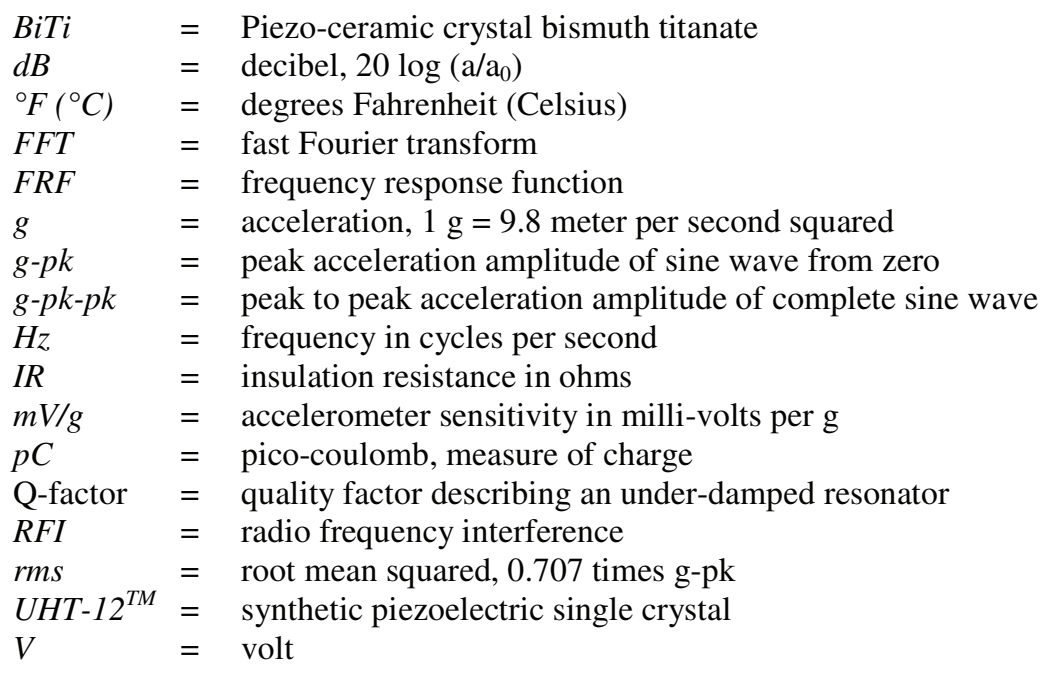

\footnotetext{
${ }^{1}$ Director, Aerospace \& Defense, 3425 Walden Ave, Depew, NY 14043, Member AIAA

${ }^{2}$ Sensoren Beratung Ensinger, Haydnallee 8, 68799 Reilingen, Germany
} 


\section{Gas Turbine Engine Monitoring Sensors}

The primary purpose of engine health monitoring accelerometers is detecting high vibration levels related to out-of-balance conditions; however, it is also possible to perform additional engine health monitoring with the data. The specific performance requirements associated with the desire for engine health monitoring ultimately determine the functional requirements (e.g., frequency response) of the accelerometer.

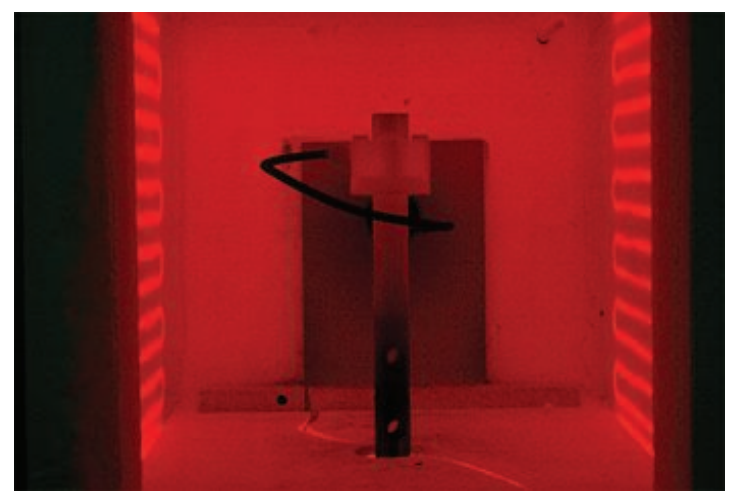

Figure 1

High-temp Accelerometer During Vibration at $1200{ }^{\circ} \mathrm{F}\left(649^{\circ} \mathrm{C}\right)$

For more than a decade the Model 357D90 shear mode accelerometer with UHT-12 ${ }^{\mathrm{TM}}$ crystals has provided successful vibration measurements on ground-based, aero derivative turbine engines (Figure 2a). The latest addition to this product family is a differential shear output version, Model EX611A20 (Figure $2 b)$.

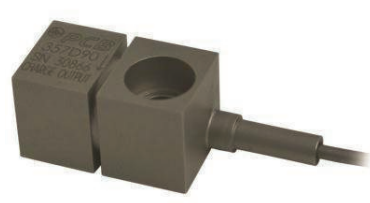

Figure 2a

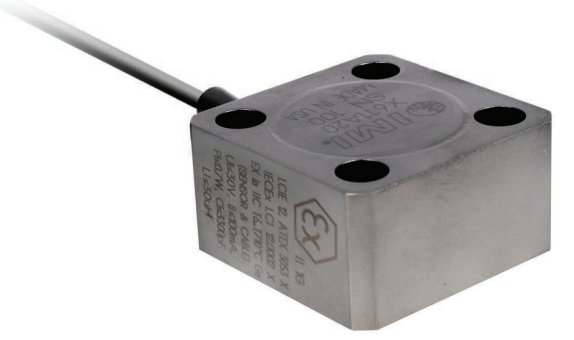

Figure 2b Shear Mode UHT-12 ${ }^{\mathrm{TM}}$ Accelerometer Designs

There are significant technical challenges involved with aero gas turbine engine vibration monitoring applications. The sensor must resolve low-amplitude, low-frequency vibration in the presence of highamplitude, high-frequency vibration, while at high operational temperatures. Engine balance readout instrumentation will integrate acceleration to velocity or displacement. When acceleration is integrated, even low levels of distortion can produce large errors in velocity and displacement signals, easily exceeding engine balance alarm levels. While analysis of time domain or FFT data may be used as diagnostic tool, it is difficult (if not impossible) to distinguish root cause from the data processed by the balance instrumentation. There are a number of potential design criteria that can cause signal anomaly at low frequency. A more detailed look at a few of these characteristics and their relationship to accelerometer performance follow. 


\section{Material Selection}

Piezoelectric sensors are made from both naturally piezoelectric crystals and artificially polarized polycrystalline ferroelectric ceramics. The choice of sensing material depends on environmental and performance requirements. Each material has unique features and advantages, which characterize its performance in various applications. Natural crystals tend to provide the highest temperature range and the lowest (or zero) pyroelectric output. However, ferroelectric ceramics offer extended frequency range and smaller size for equivalent charge output. Table 1 organizes material types ranked by temperature and pyroelectric susceptibility and thus their suitability for use in engine applications where thermal loading is not constant.

\begin{tabular}{|c|c|c|c|c|c|}
\hline \multicolumn{6}{|c|}{ Natural versus Ceramic Crystals } \\
\hline Material & $\begin{array}{c}\text { Natural Piezoelectric } \\
\text { Single Crystals }\end{array}$ & $\begin{array}{c}\text { Ferroelectic } \\
\text { Ceramic, } \\
\text { Piezoceramics }\end{array}$ & $\begin{array}{c}\text { Piezoelectric } \\
\text { Coefficient } \\
\text { pC/N }\end{array}$ & $\begin{array}{c}\text { Maximum } \\
\text { Useable Temp } \\
{ }^{\circ} \mathrm{C}\end{array}$ & Pyroelectric \\
\hline UHT-12 shear & $\mathrm{X}$ & & 12 & 650 & No \\
\hline UHT-12 compression & $\mathrm{X}$ & & 6 & 650 & No \\
\hline Quartz shear & $\mathrm{X}$ & & 4 & 250 & No \\
\hline Quartz compression & $\mathrm{X}$ & & 2.2 & 200 & No \\
\hline Tourmaline shear & $\mathrm{X}$ & & 3.5 & 650 & Yes \\
\hline Tourmaline compression & $\mathrm{X}$ & & 1.8 & 650 & Yes \\
\hline Bismuth Titanate & & $\mathrm{X}$ & 21 & 500 & Yes \\
\hline Bismuth Titanate derivates & & $\mathrm{X}$ & 14 & 600 & Yes \\
\hline
\end{tabular}

Table 1

Examples of Piezoelectric Material

Single, natural crystals, such as quartz and tourmaline, are inherently piezoelectric. Most natural occurring single crystals that are used for sensors are grown in laboratories rather than mined, resulting in consistent quality with reduced risk of supply. In addition, the man-made aspect of a natural crystal has enabled development of new, higher performance variations. The exception is tourmaline, only available through mining, and thus the supply chain is uncertain and the cost becomes prohibitive for use in sensors.

Ferroelectric ceramic materials are not inherently piezoelectric because upon chemical formulation they are in a random polycrystalline orientation. For the ceramic to become piezoelectric the individual dipoles of each crystalline structure must be aligned. The alignment process involves applying a high voltage to the material to align polar-regions within the ferroelectric ceramic element. After the artificial polarization process is complete, known as poling, the crystal may undergo a pre-aging process and then can be used in a sensor.

Ferroelectric ceramics exhibit significantly higher sensitivity or charge output per imposed unit force. A commonly used high temperature sensor material, BiTi (Bismuth Titanate), has an output three to four times its natural crystal counterpart, quartz. BiTi can be used to temperatures as high as $950{ }^{\circ} \mathrm{F}\left(510{ }^{\circ} \mathrm{C}\right)$. Various compounds may be added to the ceramic material to alter sensor characteristics but high temperature ranges come at the expense of sensitivity. Drawbacks of BiTi include the requirement for a carefully controlled environmental condition inside the sensor and for a perpetually stabilized partial pressure level of Oxygen to preserve its operational characteristics.

The new UHT-12 $2^{\mathrm{TM}}$ crystal is quite happy in any atmosphere and these sensors are backfilled with inert gas such as Argon or Nitrogen. UHT-12 $2^{\mathrm{TM}}$ crystal does not exhibit any pyroelectric output and provides for reliable operation at temperatures approaching $1200{ }^{\circ} \mathrm{F}\left(650{ }^{\circ} \mathrm{C}\right)$. While the raw charge output of this material is not as high as commonly used BiTi, additional benefits of the material include a relatively low 
capacitance and higher insulation resistance at operating temperature, which results in a low noise operation when used with a differential charge amplifier.

One often overlooked comparison of BiTi and UHT-12 is the ability to use the material in a sensing element configuration for use in a shear orientation. Physical and process limitations prevent BiTi from operating in a shear mode and thus legacy high temperature sensors are still manufactured with compression mode sensing elements. On the other hand, UHT-12 may be used in a shear configuration if properly prepared. The benefits and characteristics of these two sensing element configurations are discussed further in Section V: Mechanical Assembly.

\section{Insulation Resistance}

Very low IR (insulation resistance) may produce signal output drift in a charge amplifier; however, this is not usually a problem in a properly designed accelerometer meeting product specifications and when used with a properly designed charge amplifier. Existing BiTi engine accelerometers may have IR values around $100 \mathrm{k} \mathrm{Ohm}$ at $905^{\circ} \mathrm{F}\left(485^{\circ} \mathrm{C}\right)$, whereas the UHT- $12^{\mathrm{TM}}$ crystal will have values approximately ten times larger at the same temperature. While the noise in any charge amplified system depends on a number of factors, the larger IR value in UHT- $12^{\mathrm{TM}}$ is an important benefit, as the system noise gain is a function of the feedback resistor in the charge amplifier and the input resistance. Larger IR values reduce system noise gain. It is shown in Section VII below that the resolution of the new $10 \mathrm{pC} / \mathrm{g}$ sensor is comparable to that of a traditional higher sensitivity $50 \mathrm{pC} / \mathrm{g}$ BiTi based sensor for this reason. In addition to being a factor in determining the inherent resolution of the measurement system, the system noise gain also has the characteristic of amplifying externally imposed noise (as with RFI, for example) and therefore is an important characteristic. The new UHT- $12^{\mathrm{TM}}$ accelerometer will have a susceptibility to externally imposed noise which is approximately $8 \mathrm{~dB}(2.5 \mathrm{x})$ less than a comparable BiTi based system.

\section{Electrical Clipping Due to Axial Resonance}

Large vibration levels can over-range the amplifier electronics, resulting in an erroneous, low frequency signal. More commonly, the over-range is the result of excitation of the accelerometer's mechanical resonance. Piezoelectric accelerometers have a very high Q factor with resonant amplifications of almost $100 x$. If the excitation frequency corresponds with the accelerometer's resonance frequency, a $10 \mathrm{~g}$ signal can be amplified to 1000g and over-range the electronics. The problem can be diagnosed from observations of the spectral output, if it is observed that the low frequency error occurs at the same time as the excitation of the accelerometer's resonance. If after completing a test and evaluating the data, the test engineer observes obvious signs of problems with the data, such as the decay in baseline voltage or drops in the coherence of forced response FRF measurements, it may be a result of signal clipping.

Figure 3 shows UHT-12 ${ }^{\mathrm{TM}}$ Model EX611A00 ranged as a $100 \mathrm{mV} / \mathrm{g}$ accelerometer via a charge amplifier system. Its measured signal peaks, saturates and then exponentially decays the baseline voltage back to zero. This type of behavior indicates the input exceeded the accelerometer's measurement range (50 g-pk) of the $100 \mathrm{mV} / \mathrm{g}$ system and saturated the charge amplifier. The erroneous peak output from the $100 \mathrm{mV} / \mathrm{g}$ system indicates the peak level was $150 \mathrm{~g}$-pk and the re-ranged UHT-12 ${ }^{\mathrm{TM}}$ Model EX611A00 as a $10 \mathrm{mv} / \mathrm{g}$ accelerometer system measured data that indicates a peak level of $325 \mathrm{~g}$-pk. The lower than actual peak level measured by the $100 \mathrm{mv} / \mathrm{g}$ accelerometer is the result of the charge amplifier saturating and limiting its maximum output. The $325 \mathrm{~g}$-pk output from the $10 \mathrm{mV} / \mathrm{g}$ system was well within the specified measurement range of $500 \mathrm{~g}$-pk. 


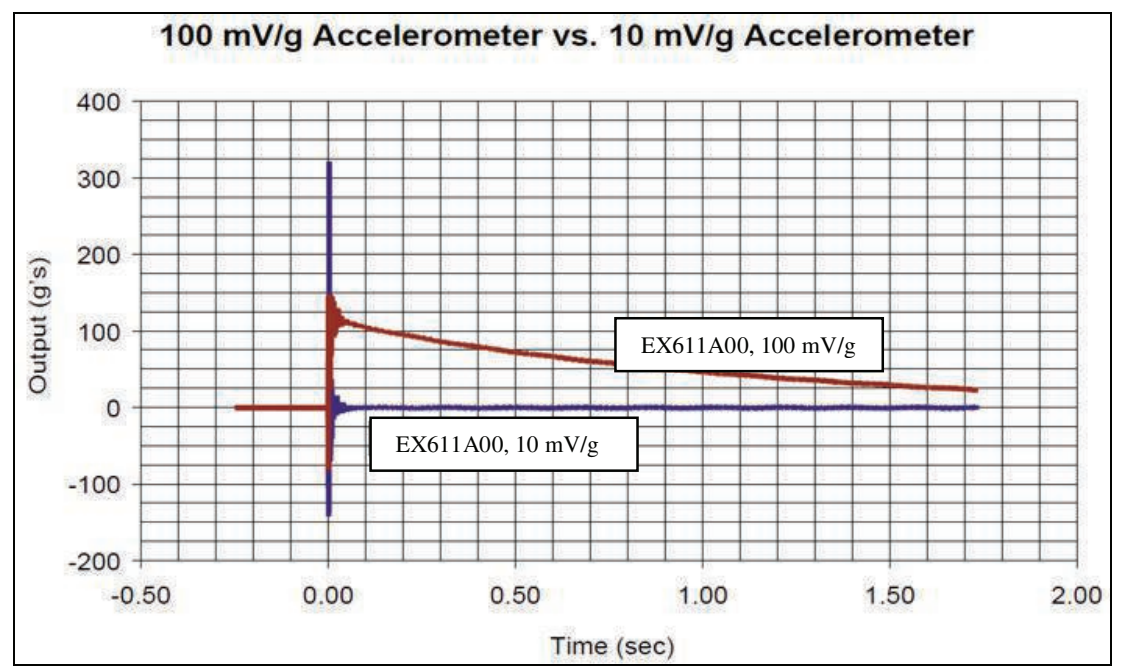

Figure 3

Accelerometer Output Signals

The expanded signals in Figure 4 are shown to illustrate how the levels from the accelerometer charge amplifier system that results in a100 mV/g sensitivity differs from the $10 \mathrm{mV} / \mathrm{g}$ system. The signal exceeds the $50 \mathrm{~g}$-pk measurement range and saturates the higher sensitivity $(100 \mathrm{mV} / \mathrm{g})$, lower amplitude range $(50$ g) accelerometer. Sometimes the signal from Figure 4 could be confused with a malfunction of the accelerometer, or even worse, not even noticed if only looking at frequency domain data.

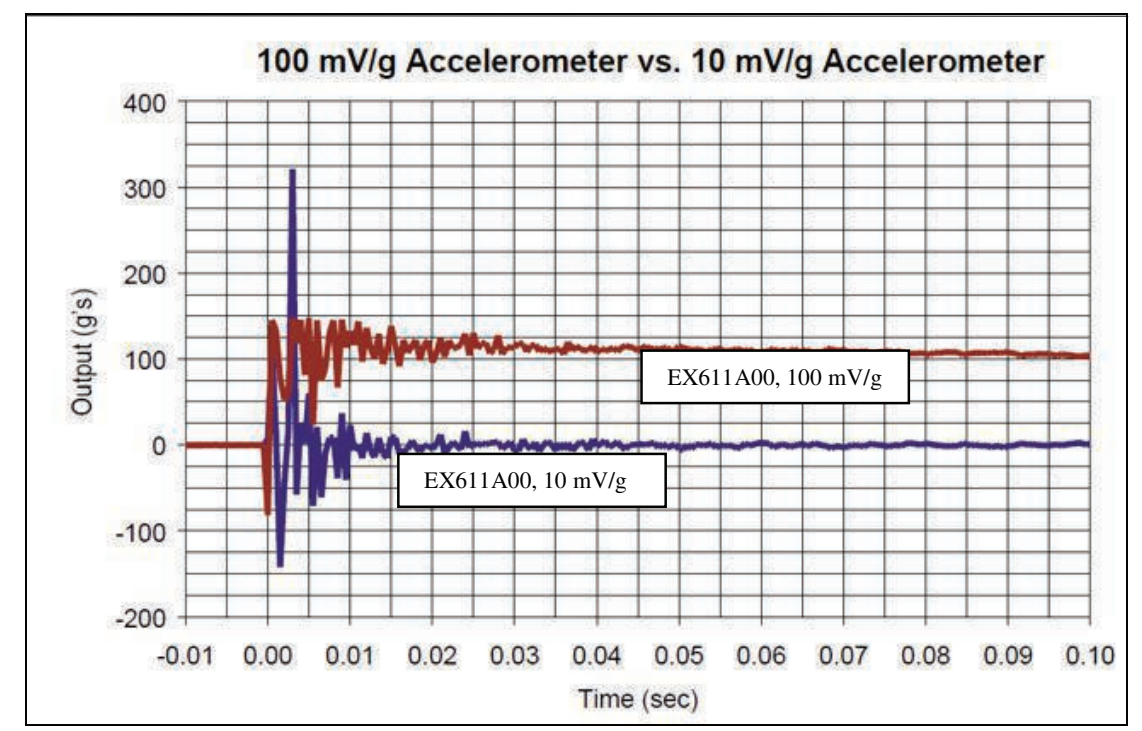

Figure 4

Accelerometer Output Signals, Expanded Time Scale

One solution to the problem is to design an accelerometer in which the axial resonance does not correspond to an engine blade pass frequency or any other natural frequency event in the engine. To accomplish this, it is very important for the accelerometer designer to work closely with the engine instrumentation system integrator. Or, since the sensor works in tandem with a charge amplifier (typically part of the Engine Monitoring Unit), it is generally good measurement practice to employ a low pass filter to the signal path. This will not only reduce the $\mathrm{Q}$ factor at resonance, but has the added benefit of extending the flat region of the sensor's useful frequency response. 


\section{Mechanical Assembly}

A natural single-crystal material can be employed in either shear or compression mode (Figure 5). A shear mode design generates charge in a shear direction vs. compression. By virtue of the configuration of the shear mode piezoelectric crystal, it is less susceptible to base strain (stress in the base that does not translate into stress in the crystals), is less susceptible to errors associated with thermal transients, is more selective in its rejection of intermodulation effects, resists off-axis stress inputs and is nearly immune to thermal characteristics.
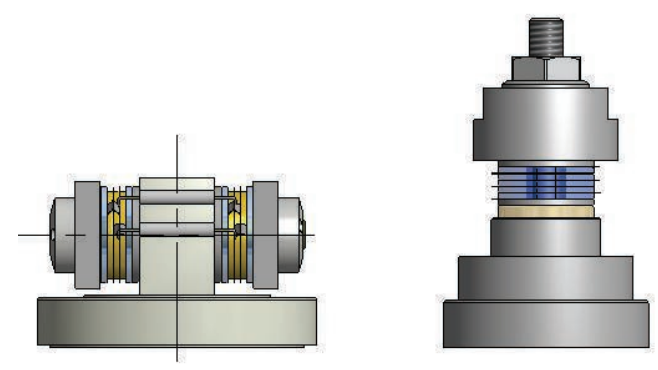

Figure 5

Shear mode element assembly (left) and compression mode element assembly (right)

\section{A. Transverse Resonance}

The blade pass frequency of a gas turbine may excite a transverse resonance of the accelerometer. Typically, the transverse resonance of shear mode accelerometer designs range from approximately $67 \%$ to $75 \%$ of the nominal axial resonant frequency; compression designs are approximately $33 \%$ of the nominal axial resonance. With transverse excitations, vibration sensitivity of the accelerometer is small and the signal output is usually small enough so as to not over-range the electronics. However, because of the high $\mathrm{Q}$ (approximately 50 in the transverse direction), low level vibration at a frequency equal to the transverse resonance will result in large motion of the seismic mass. This large amplitude motion will non-uniformly stress compression mode accelerometer design crystals, often resulting in a distorted, non-linear signal. This distorted signal will contain low frequency spectral components which fold back and will appear as false low frequency signals. In the time domain, the distortion will appear as a large number of small steps as the crystal is non-linearly stressed. If the transverse excitation is modulated by a second low frequency of say $40 \mathrm{~Hz}$, the high frequency transverse vibration signal will be erroneously modulated down to $40 \mathrm{~Hz}$ (intermodulation distortion).

In addition to having a generally higher transverse resonant frequency, the new shear accelerometer design offers two additional advantages. First, unlike the compression design, when subjected to transverse vibration, the crystal is mechanically decoupled from the region of highest bending stress as shown in Figure 6. Second, unlike the compression crystal, the shear-cut crystal responds only to the desired shear stress and does not respond to compressive, bending, and in-plane stress.

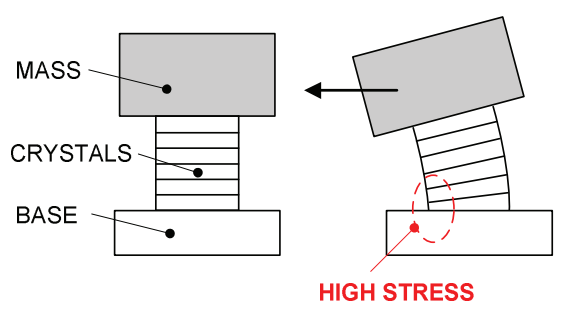

COMPRESSION DESIGN

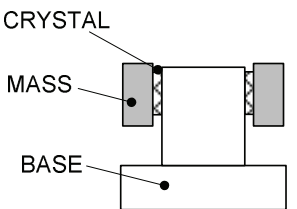

SHEAR DESIGN

Figure 6

Stress due to Transverse Motion 
Another aspect of transverse excitation occurs at frequencies other than at resonance. In turbine aircraft engines, high frequency (e.g., $13 \mathrm{kHz}$ ) blade pass excitation can reach extremely high levels (hundreds of g's rms). In such cases, transverse motion of the accelerometer's seismic mass will be large even though the $13 \mathrm{kHz}$ excitation does not correspond to an accelerometer resonance. This problem cannot be solved by altering the accelerometer's transverse resonance frequency. In a compression design, the problem is solved by paying particular attention to design aspects of the crystal stack that may improve the linearity of the accelerometer, such as: surface finish, plating, flatness, stack preload and stack concentricity. As previously indicated, the shear mode design is much more tolerant. The location of the sensing crystals and the fact they operate in a shear mode configuration inherently results in significantly less unwanted output.

\section{B. Spurious Noise Sources}

When subjected to temperature gradients, compression element assemblies (such as shown in Figure 6) will experience differential expansions/contractions of the various mating surfaces and the preload bolt. When differential expansion is large enough, the sensor may create a corresponding electrical output as stress is released and the parts instantaneously slip against each other. In addition, piezoceramic as well as tourmaline elements are pyroelectric, which means charge is created simply due to changes in temperature. In the time domain, the output from these two spurious noise sources may appear as a step output that decays at a rate governed by the signal conditioner's time constant. Example data is shown in Figures 7 for a compression accelerometer design.

The data reveals positive going spike output of approximately $13 \mathrm{~g}$ 's during the ramp up to $900{ }^{\circ} \mathrm{F}$, at time 1170 minutes. When integrated to velocity the result is $100 \mathrm{in} / \mathrm{sec} \mathrm{pk}-\mathrm{pk}$. The spiking phenomenon repeats during the cool down phase, in the negative direction with a greater rate, yet at slightly lower amplitude per occurrence.

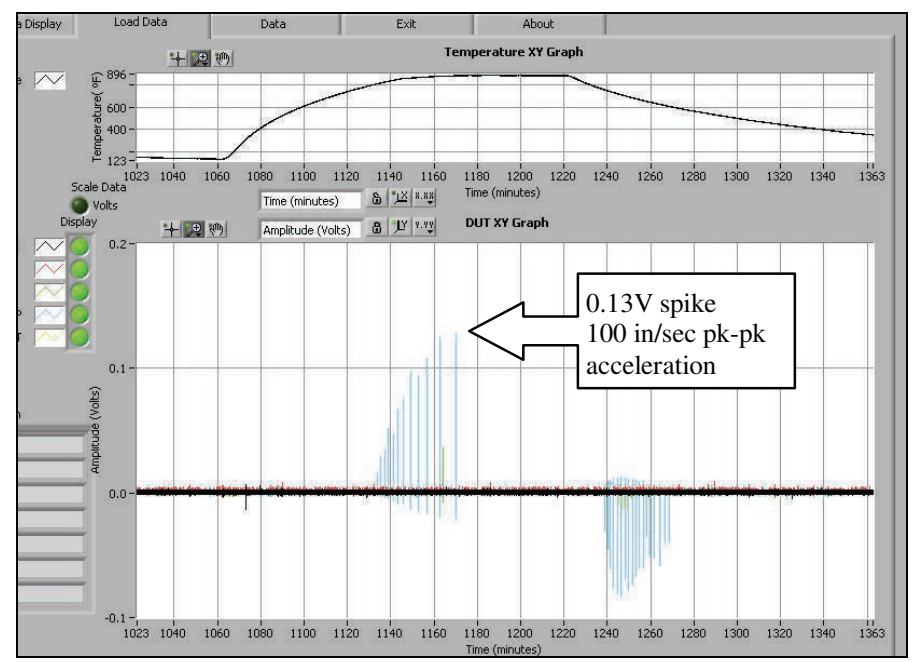

Figure 7

Typical Noise Data from Compression Mode Accelerometer During Temperature Change

The new UHT-12 $2^{\mathrm{TM}}$ shear design, which only responds to shear stress, is significantly more tolerant of thermal changes because those changes occur in the primary axis of the accelerometer, and the sensing element is oriented 90 degrees to the primary axis of vibration. The shear mode UHT-12 $2^{\mathrm{TM}}$ accelerometer consistently shows low amplitude spike levels. The fact that the UHT-12 $2^{\mathrm{TM}}$ also has no pyroelectric output is an additional advantage during thermal transient events. An example data set for the thermal response of the new UHT- $12^{\mathrm{TM}}$ shear design is shown in Figure 8. 


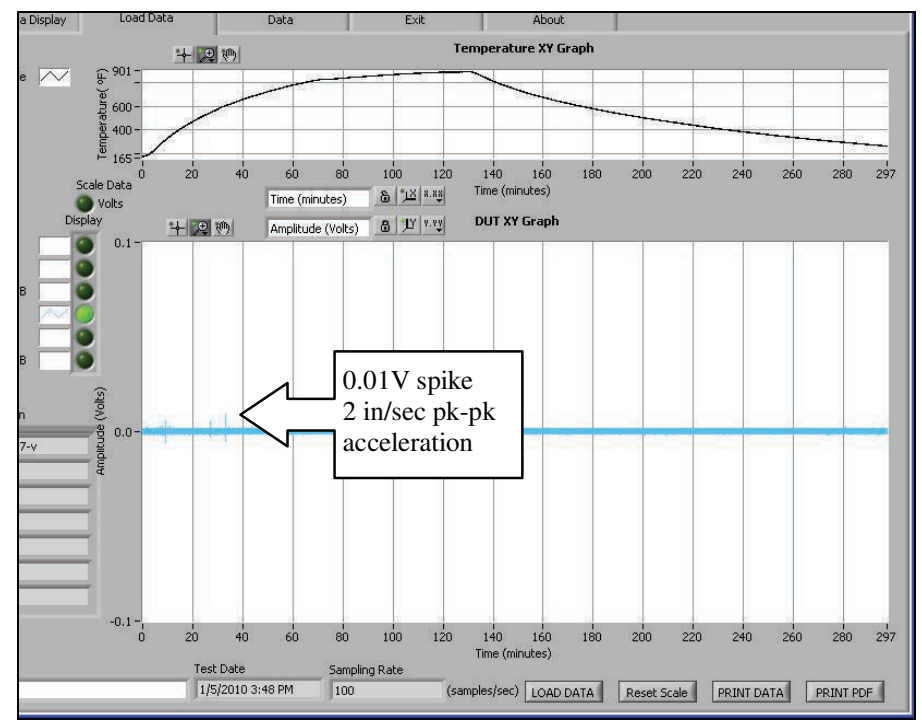

Figure 8

Typical Noise Data from Shear Mode Accelerometer During Temperature Change

To engine balance instrumentation, a step output from an accelerometer will look like a large low frequency signal. The problem occurs during or soon after a change in temperature, such as going from idle to full power for take-off.

\section{Pyroelectric Affects}

There are numerous temperatures, bandwidth, mounting and other tradeoffs that must be considered in high-temperature applications. Temperature effects exist beyond the limitation of the operating environment. Temperature changes may produce extraneous outputs and may change the sensitivity and other parameters. Piezoelectric sensors cannot produce an output in response to a constant temperature, as they cannot produce an output in response to any constant input. However, they can produce an output in response to a change in temperature.

Some piezoelectric materials often behave in a pyroelectric manner and generate an output in response to temperature gradients. Thermal expansion related to these temperature gradients tends to change pre-load stresses on the crystal-to-sensor assembly and further aggravates the sensor output. Sometimes piezoelectric sensors can show sharp spikes in their output after a large temperature change that can be attributed to electrostatic surface discharge of pyroelectric fields. Spikes which continue after a pyroelectric discharge can be related not only to the piezoelectric material but to the design of the individual components and processing of the sensor.

A design that utilizes ferroelectric ceramic material in compression mode will have a greater pyroelectric output than that of a piezoelectric shear design or one using a natural crystal because of two effects. First, the piezoelectric material in compression mode accelerometers is directly coupled to the environment through the base of the sensor. Second, the ferroelectric material is sensitive to uniform temperature changes on those surfaces perpendicular to the axis of polarization.

Pyroelectric output is a low frequency phenomenon that is typically well below frequency ranges of interest. It is possible to reduce its affects with the use of high-pass filtering within measurement system electronics. 


\section{Signal to Noise Ratio}

Although the traditional BiTi produces a sensitivity of $50 \mathrm{pC} / \mathrm{g}$ the UHT- $12^{\mathrm{TM}}$ crystal provides between 5 and $10 \mathrm{pC} / \mathrm{g}$ and has proven suitable for turbine vibration measurements. This lower sensitivity provides a further opportunity for smaller parts, lighter weight and wider frequency response. In today's world of low noise electronics and high dynamic range analog to digital converters, there is simply no need for a larger, higher sensitivity element. In fact, when the UHT-12 $2^{\mathrm{TM}}$ element, which has lower element capacitance and higher insulation resistance than traditional BiTi sensors, is coupled with a suitable differential charge amplifier, a standardized $25 \mathrm{mV} / \mathrm{g}$ output (resulting in a $+/-5 \mathrm{~V}$ swing at $+/-200 \mathrm{~g}$ 's) is provided. The dynamic range is greater than $120 \mathrm{~dB}$, providing a measurement resolution typically better than $0.002 \mathrm{~g}$ 's (broadband from $1 \mathrm{~Hz}$ to $10,000 \mathrm{~Hz}$ ).

The two plots in Figure 9 show actual noise data taken with two charge amplifiers, each having a low frequency response of $1.6 \mathrm{~Hz}(-3 \mathrm{~dB})$. One of the amplifiers was configured to provide a $25 \mathrm{mV} / \mathrm{g}$ output with the proposed sensor, and the other was configured to provide a $25 \mathrm{mV} / \mathrm{g}$ output with a traditional 50 $\mathrm{pC} / \mathrm{g}$ BiTi based sensor.
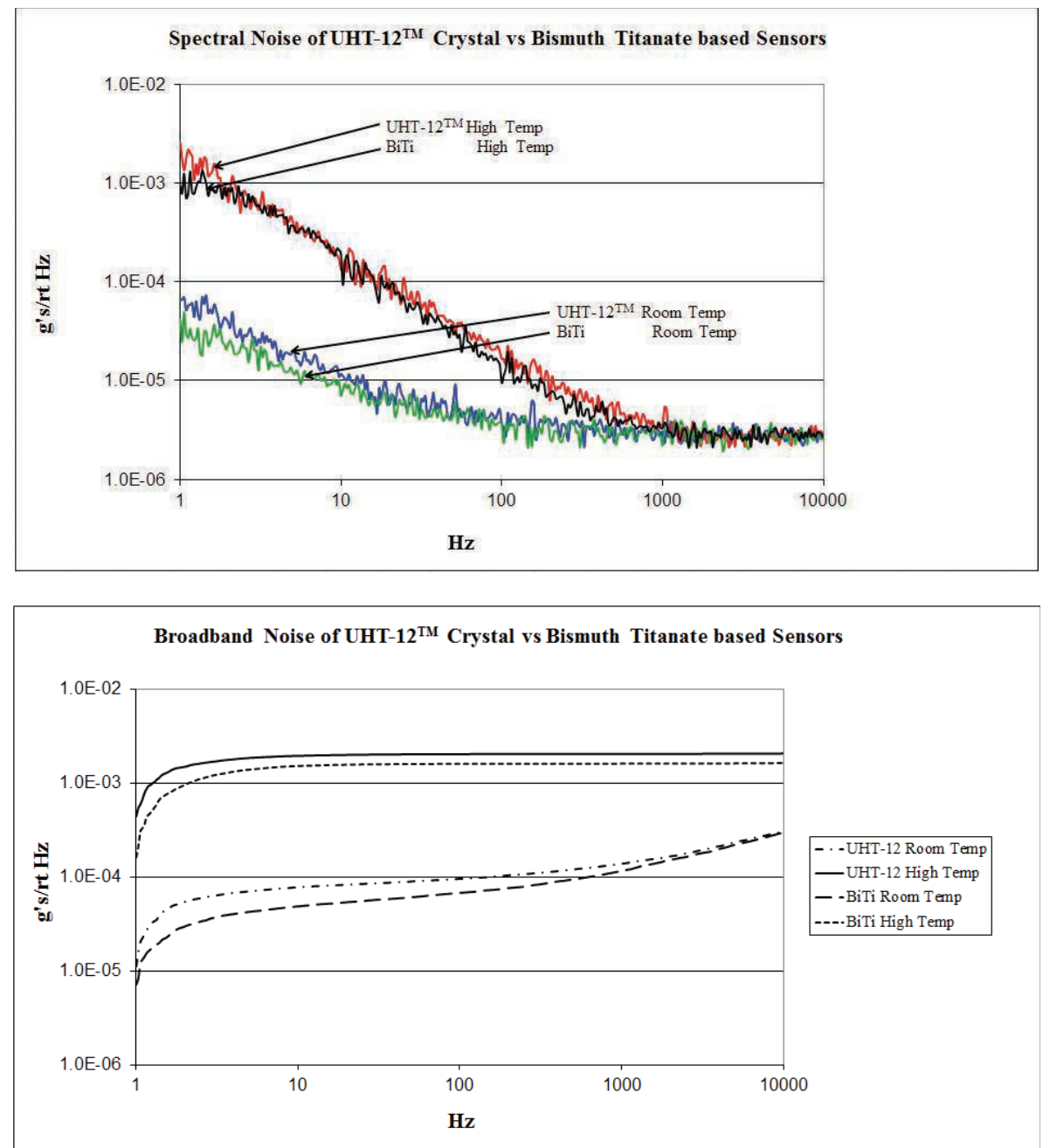

Figure 9

Noise Comparison of UHT-12 ${ }^{\mathrm{TM}} 10 \mathrm{pC} / \mathrm{g}$ Accelerometer vs. BiTi 50 pC/g

Two configurations of each sensor were compared: one with very high insulation resistance (such as the sensors would exhibit at room temperature) and one with low insulation resistance (such as each sensor would exhibit at $485^{\circ} \mathrm{C}$ ). It clearly can be seen that the reduction in insulation resistance increases system 
noise in both cases. Note however, that the system resolution is comparable for the two systems. Both have a noise floor of approximately $2 \mathrm{mg}$ 's (against the stated spec of $10 \mathrm{mg}$ 's) despite the fact that the new UHT- $12^{\mathrm{TM}}$ accelerometer has a charge output that is $1 / 5$ that of a BiTi based sensor.

\section{Conclusion}

Both sensor theory and actual on-engine data support the use of high temperature shear technology as the preferred measurement solution over the legacy design of compression accelerometers. The use of commercially available, synthetic, naturally piezoelectric crystals (UHT-12 ${ }^{\mathrm{TM}}$ ) provides the added benefits of higher temperature operation, no pyroelectric output, higher insulation resistance, better long term reliability and long term commercial sourcing security. Note that the UHT- $12^{\mathrm{TM}}$ material also permits the use of a shear sensing element configuration. High temperature BiTi materials are not able to function in a shear configuration.

Several lessons have been learned during the development of UHT-12 ${ }^{\mathrm{TM}}$ shear mode, high temperature accelerometers, versus compression mode designs.

1. Shear mode versus compression mode designs have less measurement error

2. Symmetrical geometry of a bolted shear configuration minimizes nonlinearities in output, especially at sensor resonances

3. Use of inert gas fill minimizes contamination issues and oxidation of internal parts

4. Methods and designs relating to bolt pre-stretching of sensing crystal element stacks provides for consistent preload, considering temperature and vibration extremes

5. Selecting appropriate super alloy metals minimizes sensing crystal element stack growth at temperature for a more uniform preload

6. Employing non-pyroelectric crystal materials minimizes thermal errors

7. Employ synthetic crystal materials such as UHT-12 ${ }^{\mathrm{TM}}$ versus BiTi for improved long-term stability and no possible reduction of the crystal material from lack of Oxygen

8. Employ synthetic crystal materials such as UHT- $12^{\mathrm{TM}}$ versus BiTi to extend high end sensor temperature ranges (phase transition point is greater than $2600^{\circ} \mathrm{F}\left(1427^{\circ} \mathrm{C}\right)$ )

Whether used in aircraft turbine engines, rocket motors, or power generation stations, these accelerometers must provide high levels of accuracy, stability and reliability. Therefore these instruments used in extreme high temperature environments require special consideration during the design and manufacturing process.

\section{Acknowledgments}

The author thanks PCB Piezotronics Inc. Vice President of Engineering, Mr. Dave Lally and Lead Design Engineer, Mr. Nick Fulciniti, for their tireless support in providing measurements and advice in preparation for this paper. A special thanks also to Mr. Hans Ensinger, from Sensoren Beratung Ensinger, Reilingen, Germany for the inspiration to put our many conversations down on paper.

(C) 2014 PCB Piezotronics, Inc. 